\title{
Liame
}

Histoire et histoire de l'art des époques moderne et contemporaine de l'Europe méditerranéenne et de ses périphéries

$24 \mid 2012$

Territoires urbains en construction dans l'espace méditerranéen à l'époque moderne

\section{Topographies de « minorités »}

Notes sur Livourne, Marseille et Tunis au XVII ${ }^{\mathrm{e}}$ siècle

\section{Guillaume Calafat}

\section{(2) OpenEdition}

1 Journals

Édition électronique

URL : http://journals.openedition.org/liame/271

DOI : 10.4000/liame.271

ISSN : 2264-623X

Éditeur

CRISES - Centre de Recherches Interdisciplinaires en Sciences Humaines et Sociales de Montpellier

Référence électronique

Guillaume Calafat, «Topographies de «minorités » », Liame [En ligne], 24 | 2012, mis en ligne le 10 juillet 2013, consulté le 19 avril 2019. URL : http://journals.openedition.org/liame/271 ; DOI : 10.4000/ liame.271

Ce document a été généré automatiquement le 19 avril 2019

Licence Creative Commons

Les contenus de Liame sont mis à disposition selon les termes de la Licence Creative Commons

Attribution - Pas d'Utilisation Commerciale - Pas de Modification 4.0 International. 


\title{
Topographies de « minorités »
}

\author{
Notes sur Livourne, Marseille et Tunis au XVII ${ }^{\mathrm{e}}$ siècle \\ Guillaume Calafat
}

1

2 L'historiographie sur les étrangers sous l'Ancien Régime ne cesse de rappeler l'importance du droit et des statuts dans les processus d'inclusion ou d'exclusion des groupes minoritaires - entendus ici à la fois comme des groupes de populations numériquement et juridiquement en situation de "minorité». Ainsi, la question des privilèges de citoyenneté et de droit d'accès à la propriété se trouve, plus largement, au cœur des débats concernant la construction de la société civile et la genèse de l'État moderne ${ }^{1}$. Bien entendu, l'histoire de l'espace urbain ne peut faire l'économie de ces questions, dans la mesure où le bâti est souvent un bon indicateur des processus de négociation juridique et politique qui contribuent à l'installation des «étrangers » dans les villes de l'époque moderne ${ }^{2}$. Certes, la variété des formes d'intégration en ville, plus ou moins complète et possible selon les zones de provenance et les sociétés dites "d'accueil», de même que le caractère particulièrement insaisissable de la catégorie «étranger» sous l'Ancien Régime interdisent toute conclusion systématique ${ }^{3}$. Pour paraphraser Bernard Lepetit « le spatial n'explique pas le juridique », mais force est bel et bien de constater que "parcours juridiques et parcours spatiaux sont à envisager ensemble $»^{4}$. En cela, la morphologie urbaine et les traces laissées par les populations «étrangères " dans les villes de l'époque moderne s'avèrent un complément utile à l'histoire juridique et sociale des « minorités ».

Le choix de l'espace méditerranéen comme observatoire ne saurait traduire l'existence d'un « modèle », dont il faut sans aucun doute se défier sous peine de surévaluer les effets d'une supposée unité du monde méditerranéen, ou encore d'extrapoler des réalités urbaines et sociales partagées bien au-delà de la Mer Intérieure ${ }^{5}$. Chercher, en effet, une forme urbaine spécifiquement méditerranéenne relève tout au mieux du vœu pieux, quand la quête n'est pas tout simplement teintée d'a priori culturalistes ${ }^{6}$. De même, l'établissement de typologies ou de comparaisons transméditerranéennes pose nécessairement le problème périlleux d'utilisation de catégories d'analyse et d'« autorités analogiques " inadaptées ${ }^{7}$. En choisissant de mettre en regard Marseille, Tunis et 
Livourne au XVII ${ }^{\text {e }}$ siècle, il s'agit plutôt d'observer différents modes de territorialisation de la présence étrangère, trois manières possibles de la rendre visible à l'échelle urbaine. La variété des morphologies urbaines - qui traduit bien souvent des asymétries juridiques et des différences de statuts - ressort avec force dans la mesure où le triangle MarseilleLivourne-Tunis compose un espace commercial étroitement interconnecté, au sein duquel circulent tout à la fois marchandises, informations, capitaux et personnes aux $\mathrm{XVII}^{\mathrm{e}}$ et XVIII ${ }^{\mathrm{e}}$ siècle ${ }^{8}$.

\section{Espaces portuaires et lieux de transbordement}

Marseille, Livourne et Tunis (en l'occurrence le binôme Tunis-La Goulette) sont trois villes portuaires, c'est-à-dire des sites aménagés pour abriter des navires et permettre le transbordement de marchandises. Cette première définition suppose des infrastructures d'accueil importantes (fortifications et forteresses, digues, quais, darses, magasins et lazarets - même si celui de l'île de Chikly, au large de la Goulette semble tomber en désuétude au XVII ${ }^{e}$ siècle ${ }^{9}$ ). "Place permanente", le portus est à la fois un lieu clos, protégé, utilisé comme entrepôt ou étape pour le chargement et le déchargement de marchandises, comme une zone de transit, d'entrée, de passage ${ }^{10}$. À la fois lieu du mouvement et point de contrôle, Marseille, Livourne et Tunis sont des villes-frontières, des interfaces où les autorités locales surveillent le flux des migrations temporaires ou permanentes et contrôlent les marchandises ${ }^{11}$. Dans la Régence de Tunis, par exemple, les bateaux qui transportent des biens ne peuvent transiter que par des lieux surveillés par des postes de douane (Tunis, Bizerte, Sousse, Sfax au début du XVII ${ }^{e}$ siècle, et plus tard Porto-Farina, Monastir et Djerba), sous peine d'encourir saisie des biens et absence de protection consulaire ${ }^{12}$. Les formalités juridiques créent ainsi des parcours spatiaux obligés, même si le débarquement clandestin de marchandises sur les plages ou les transbordements entre bateaux demeurent des pratiques courantes. Le port combine deux fonctions: d'une part, celle de place de commerce ouverte pour favoriser les échanges; de l'autre, celle d'espace frontalier, qui suppose une armature institutionnelle dense visant à contrôler les migrants et leurs effets.

\section{Marseille}

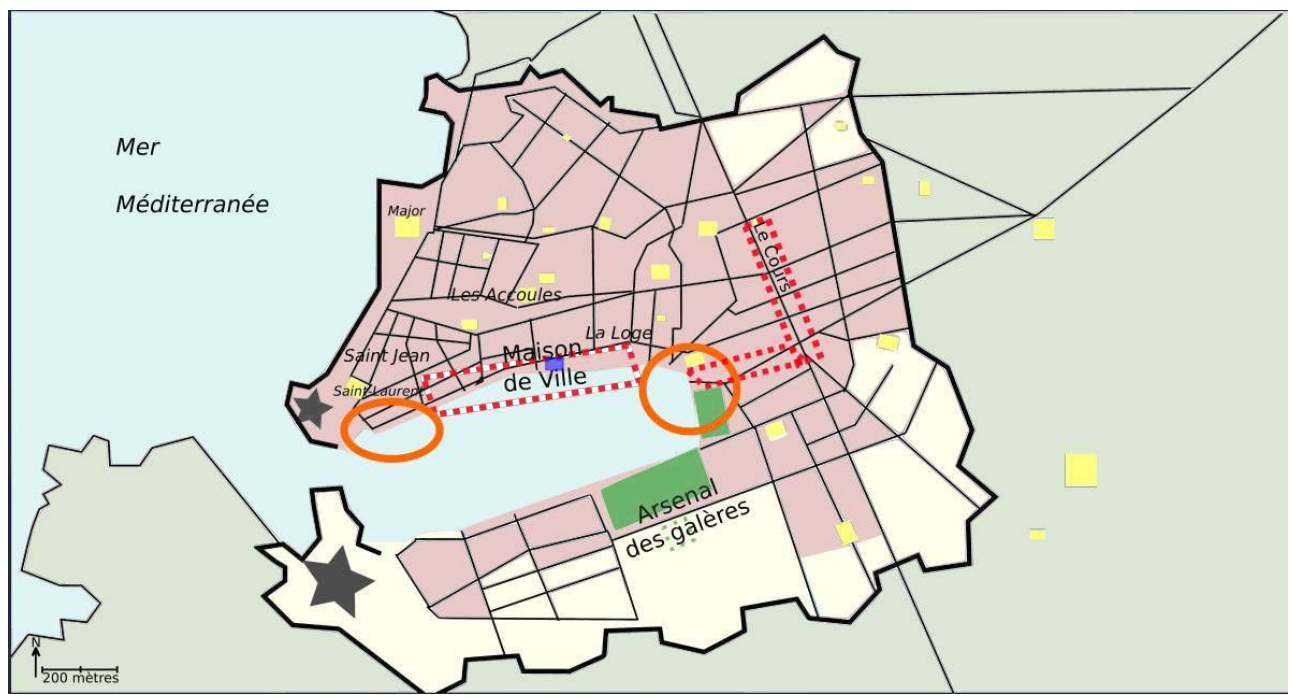




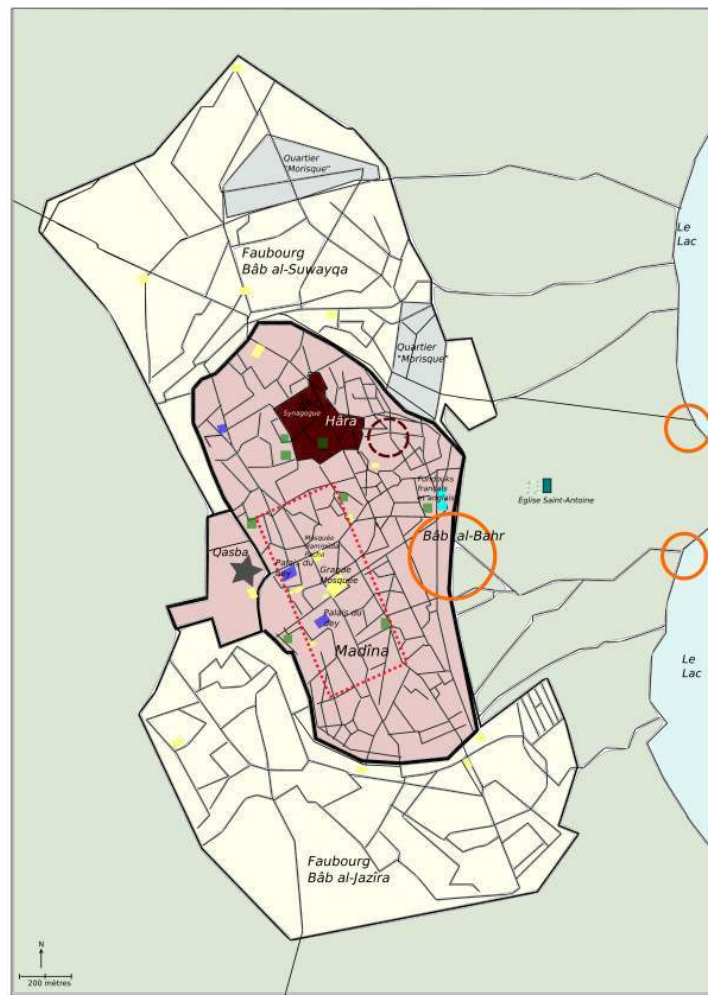

5 Marseille et Tunis comptent toutes deux des « quartiers étrangers ", entendus comme des secteurs de la ville marqués par une forte proportion de personnes de diverses origines. À Marseille, le quartier Saint-Jean, au sud-ouest de la ville, à l'entrée du port, est un lieu « où habitent presque tous les pêcheurs et hommes de $\operatorname{mer}^{13}$ ». À Tunis, la zone qui s'étend dans les environs de Bâb al-Bahr (la porte de la Mer), à l'est de la ville, et qui débouche sur le lac par lequel s'établissaient les relations entre Tunis et le port de la Goulette abrite un grand nombre de marchands et de marins européens. Français, Anglais puis Hollandais y établissent à partir de la fin du XVI ${ }^{\mathrm{e}}$ siècle leurs consulats - au Moyen Âge, la même zone avait été investie par les marchands pisans, génois, majorquins et aragonais $^{14}$. On installe à l'extérieur des portes des cimetières pour les catholiques, à côté de l'église Saint-Antoine, puis, à la suite des demandes répétées du consul anglais, un cimetière pour les protestants en 1645. Bâb al-Bahr devient le « quartier franc » de Tunis, un quartier de transbordement directement relié au lac de Tunis et à la Goulette - un quartier tenu à bonne distance de la Qasba et du quartier résidentiel des élites autour de la Grande Mosquée ${ }^{15}$. Le chevalier d'Arvieux qui passe à Tunis en 1668 la décrit comme « un pays de liberté » où « la Religion n(e) gêne personne ${ }^{16}$ ». Juifs et chrétiens jouissent d'une relative liberté de culte dans la capitale de la Régence, marquée de surcroît, au début du XVII ${ }^{e}$ siècle, par l'arrivée de populations allogènes, morisques et européennes ${ }^{17}$. 


\section{Livourne}

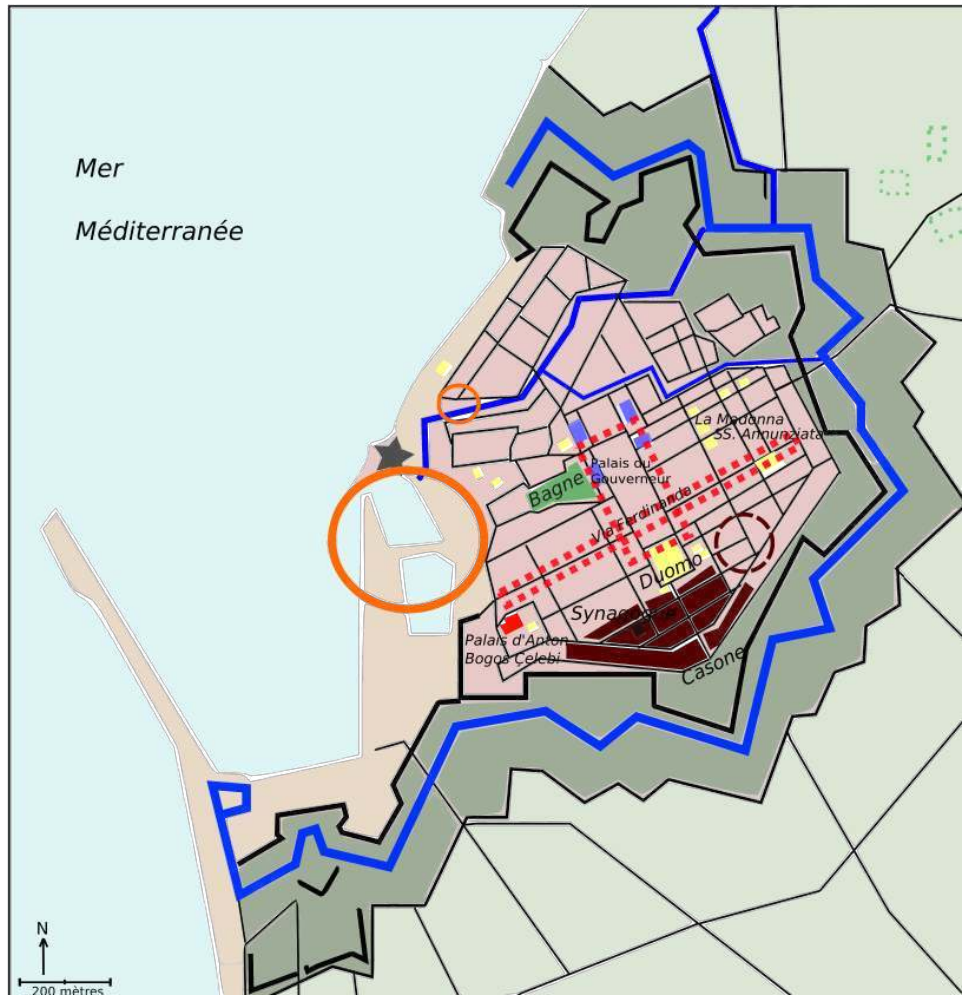

\section{Légende :}

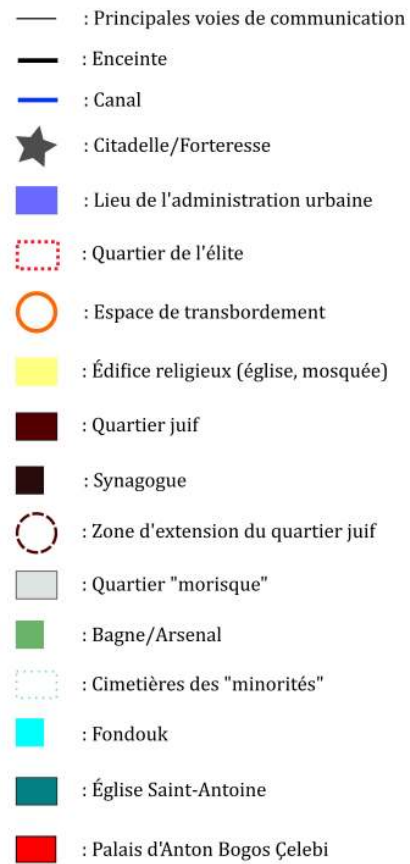

6 À la différence des "antiques » Marseille et Tunis, Livourne est une "ville nouvelle ", fruit du projet économique et militaire médicéen amorcé au XVI ${ }^{e}$ siècle visant à faire d'un petit port du littoral tyrrhénien le pendant portuaire de Florence et de Pise, ville à laquelle elle est reliée depuis la seconde moitié du XVI ${ }^{e}$ siècle par le Canale dei Navicelli. Ce 
projet se traduit par la construction d'une cité fortifiée à plan pentagonal, selon les canons de la trattatistica architecturale du temps ${ }^{18}$. En 1591 et 1593, le grand-duc Ferdinand $\mathrm{I}^{\mathrm{er}}$ de Médicis accorde franchises, immunités et privilèges aux marchands « de toutes nations » qui viendraient s'installer à Pise et à Livourne ${ }^{19}$. Le port, peu peuplé à la fin du XVI ${ }^{\mathrm{e}}$ siècle, connaît une croissance démographique exceptionnelle : la ville compte 570 habitants en 1590, plus de 12000 en $1642^{20}$. Dès les années 1610 , Livourne devient progressivement le centre de gravité économique de la Toscane maritime ${ }^{21}$. L'accroissement démographique et le développement économique du port toscan font l'admiration des contemporains, quoique les nouveaux habitants de Livourne n'aient guère bonne réputation ${ }^{22}$. Les récits de voyage ou les manuels à l'usage des marchands insistent sur le brassage qui existait - à différents degrés - dans les trois ports méditerranéens. Au début de l'année 1690, Jean Dumont décrit Marseille comme «l'une des [villes les] plus peuplées de France ", mais il exagère sans doute quand il ajoute qu'il y a «le «tiers d'étrangers ${ }^{23}$ ». Dans The Merchants Mappe of Commerce, qui offre en 1638 un large panorama géographique des marchés de l'époque moderne, Lewes Roberts explique qu'il a résidé à Marseille et voyagé à plusieurs reprises à Livourne : son expérience des deux places de commerce, censée donner du crédit à ses observations, l'amène à les qualifier de «free cities ${ }^{24}$ ». Ces «villes franches» ou "ports francs» le sont, sous sa plume, du point de vue douanier: Livourne marque à la fois les contemporains par la liberté de culte qui y règne, mais aussi parce que l'on ne peut y être poursuivi pour des dettes contractées dans d'autres pays ${ }^{25}$. La seconde moitié du XVII ${ }^{e}$ siècle à Marseille est, quant à elle, marquée par la subordination du droit municipal au droit du royaume, traduite spatialement par l'érection du fort Saint-Jean, symbole de la puissance royale victorieuse des révoltes urbaines et de la Fronde ${ }^{26}$. Avec l'édit de port franc de 1669, les étrangers semblent davantage échapper au contrôle direct des notables marseillais ${ }^{27}$. La durée de résidence et la maison font désormais partie des conditions d'obtention de la naturalité, d'où découle le droit de bourgeoisie ${ }^{28}$.

\section{Les espaces des nations}

$7 \quad$ Les communautés étrangères dans la plupart des grandes places de commerce et dans les espaces portuaires de Méditerranée s'organisent depuis le Moyen Âge en "nations ", c'est-à-dire en communauté de provenance (d'une ville, d'un «État») dotée d'une personnalité juridique, et généralement pourvue d'un représentant, un «consul». Ce système permet l'existence de relais et de réseaux de solidarité entre les minorités habitant sur place, les commerçants de passage et les autorités locales. La reconnaissance juridique des «nations » s'appuie sur des traités entre pays hôtes et autorités politiques de la nation considérée, ou bien sur une base contractuelle entre les autorités locales et les représentants d'une communauté étrangère déjà installée ou en voie d'installation. L'organisation en «nations» peut avoir des manifestations spatiales aisément identifiables dans l'espace urbain: hôpitaux, cimetières, "scuole, églises 'ethniques', fondaci [...], palais aristocratiques", autour desquels se construisent les réseaux de sociabilité «nationaux », constituent autant de symboles de la présence de communautés « étrangères » dans le port ${ }^{29}$. À Marseille et à Livourne, les consuls des nations étrangères vivent dans des maisons spacieuses et bien situées, près des lieux du pouvoir municipal et à proximité du port, pour être accessibles aux capitaines, aux marchands et aux voyageurs étrangers. Issus la plupart du temps des milieux du négoce, les consuls et leurs 
commis arpentent la ville : ils sont présents à la Loge, dans les assemblées de marchands, sur les quais pour voir arriver les navires et obtenir des informations sur le trafic, la conjoncture et les éventuels conflits. La maison des consuls, qui sert de chancellerie, est également considérée comme« le lieu le plus propre pour [...] exposer au public» les ordonnances, règlements royaux et autres décisions ou nouvelles importantes ${ }^{30}$.

Il est peu aisé de cerner des espaces «nationaux » dans la Marseille du XVIIe siècle, la dissémination des "étrangers" semblant prévaloir. La "nation" juridique ne semble apparemment pas y façonner d'espaces minoritaires, et la distribution des "étrangers " dans la ville paraît davantage le produit de la stratification sociale ${ }^{31}$. En outre, pour les étrangers qui professent la même religion que la société d'accueil - à l'exception des plus pauvres ou des migrants saisonniers - le statut d'étranger est transitoire. Les étrangers catholiques peuvent, à Marseille et à Livourne, devenir des sujets du roi ou du grand-duc et prétendre aux hautes charges municipales, la richesse jouant le rôle d'accélérateur d'inclusion. L'inclusion est encore plus nette à Tunis, où des convertis à l'Islam, génois ou corses, atteignent le sommet du pouvoir politique et économique à l'échelle de la Régence tout entière en devenant beys ou deys ${ }^{32}$. Précisons cependant que des processus d'agrégation sont observables : les Grecs de Livourne s'installent progressivement autour de l'église de la Santissima Annunziata, inaugurée en 1606 pour les Grecs catholiques. Au milieu des années 1620 , près de trente familles vivent dans les rues adjacentes : « via delle Galere », « via dei Cavalieri » et dans la rue appelée « via dietro alla chiesa de Greci »33. En faire un «quartier grec » serait toutefois réducteur, puisque l'on y trouve, par exemple, nombre de Français ${ }^{34}$.

9 La religion demeure un facteur déterminant d'inclusion ou d'exclusion socio-spatiale des «étrangers ». L'étranger catholique à Marseille, marchand et aisé, a la possibilité de suivre un parcours d'intégration balisé : pour accéder au droit de bourgeoisie, qui, dans les «bonnes villes » de l'Ancien Régime, confère une notabilité sociale et politique et surtout - exempte du droit d'aubaine, il faut dans la première moitié du siècle «prouver un séjour ininterrompu de dix ans, avoir acquis des immeubles et créé un établissement commercial ${ }^{35} »$. Ce droit de bourgeoisie (ou citadinage), comme dans tout le bassin occidental de la Méditerranée, permet de jouir d'un bien plus grand prestige social que la naturalité. Ainsi, les voisins catholiques (Italiens, Génois et Savoyards au premier chef, mais aussi Corses et Espagnols), accèdent aux charges publiques et jouent un rôle de premier plan dans la vie économique locale. Ces étrangers-là « marquent l'espace de leur éclat, tenant maison ouverte, offrant fêtes et feux d'artifice, fontaines et aménagements de places ${ }^{36} »$; ils logent dans les maisons cossues du quartier de la Loge, des Accoules et de l'Hôtel de Ville ${ }^{37}$. Le modèle municipal marseillais vis-à-vis des «étrangers » apparaît donc comme un modèle d'intégration horizontale et de mise en conformité, qui passe d'ailleurs bien souvent par des alliances matrimoniales avec les principales familles de notables provençau ${ }^{38}$. En cela, les autorités locales tendent à privilégier la « fiction de l'homogénéité de l'espace urbain ${ }^{39} "$, censée notamment traduire l'unité et l'harmonie religieuse de la ville provençale. L'espace des minorités n'est quasiment pas négociable dans la Marseille du XVII ${ }^{\mathrm{e}}$ siècle, dans la mesure où le statut d'étranger est pensé soit en termes de domination économique et sociale (droit d'aubaine, interdiction d'accès aux charges municipales et aux fermes lucratives...), soit comme un statut temporaire avant la bourgeoisie ou la naturalisation, qui oblige à une forme d'adéquation sociale - et spatiale - avec les pratiques locales. 
10 Au début des années 1620, les Arméniens font ainsi les frais des entraves mises par la municipalité et la chambre de commerce de Marseille à l'établissement des marchands originaires de la Nouvelle-Djoulfa, faubourg d'Ispahan : le conseil de ville, en effet, prend notamment la décision en novembre 1621 d'interdire aux capitaines de navires français « de conduire et d'enlever Ponant et Levant aulcuns marchands arméniens ou persiens ou facultés d'iceuls, pour les transporter à marseille ", sous peine d'une lourde amende et d'une confiscation des biens meubles et immeubles ${ }^{40}$. Ces derniers ont dès lors tendance à recourir à des prête-noms ou à privilégier l'établissement à Livourne et à Gênes. À partir des années 1660, ils bénéficient des mesures attractives de Colbert et forment une petite colonie prospère de marchands spécialisés dans l'indiennage ${ }^{41}$. Les protestants allemands, hollandais ou anglais à Marseille et à Livourne (jusqu'en 1707) n'ont, quant à eux, pas d'espace dévolu à leurs cultes et se dissimulent dans des logis privés; à Livourne cependant, certains prédicateurs anglicans abordent dans le port, non sans susciter le «scandale» des autorités locales et de l'Inquisition ${ }^{42}$, montrant ainsi les limites de l'adresse cosmopolite qui introduit les lois Livornine. Être étranger ne signifie donc pas du tout la même chose si l'on est catholique ou protestant et les parcours spatiaux diffèrent en conséquence: les Français catholiques à Livourne se rendent majoritairement au Duomo le dimanche, quand les Français issus des milieux protestants vont prier dans la Chiesa della Madonna, jésuate, où se rassemblent Anglais, Hollandais, Nouveaux Chrétiens puis Arméniens ${ }^{43}$. La géographie portuaire joue également un rôle, dans la mesure où nombre des cérémonies religieuses des protestants anglais (baptêmes et messes) peuvent avoir lieu sur les bateaux et non sur le sol toscan ${ }^{44}$.

11 L'espace de la minorité religieuse est davantage délimité et peut regrouper de manière temporaire des communautés de provenances diverses : l'église grecque de la Santissima Annunziata accueille aussi des Melchites à Livourne ${ }^{45}$. De même, les Maronites, les "Turcs de nation», certains esclaves de longue durée, les Arméniens et plus généralement les voyageurs curieux se rendent fréquemment dans les bains de la maison $\mathrm{du}$ richissime Anton Bogos Çelebi, originaire de Smyrne et établi à Livourne dans la seconde moitié du XVII ${ }^{e}$ siècle ${ }^{46}$. D'espace privé, la demeure de Çelebi était devenue un espace semi-public, proche de la darse et des quais, où les voyageurs et les habitants vêtus «à la turque " pouvaient se retrouver. La «nation» recoupe ainsi une pluralité de parcours spatiaux, qui dépendent bien souvent de l'obédience. Il n'était pas rare - tout particulièrement à Livourne - qu'il y ait des minorités religieuses au sein même de la minorité « nationale » qui servait alors de paravent. Quand ils n'ont pas de quartiers ou de lieux clos dévolus, cela contraint les « étrangers minoritaires » à des regroupements temporaires, les moins ostentatoires possibles et permettant la dissimulation.

\section{Les espaces clos. Fondouks et bagnes}

12 Tunis, Livourne et Marseille disposent au XVII ${ }^{e}$ siècle d'espaces clos dévolus aux « étrangers ». À Tunis, on pense bien entendu aux bâtiments connus, sur le pourtour de la Méditerranée, sous le terme grec arabisé de « fondouks ». Construits vraisemblablement à la fin des années 1650 grâce à une concession du Divan et sous le règne de Hammûda Pacha, les marchands européens ont à leur disposition deux fondouks : celui des Français (pour les catholiques), et celui des Anglais et des Hollandais (pour les protestants) ${ }^{47}$. Situés dans la partie basse de la Madîna, ces édifices adoptent la forme des khans et des caravansérails locaux, et s'inscrivent dans la droite ligne des fondouks médiévaux italiens 
et catalans sous les Hafsides ${ }^{48}$. Le fondouk des Français, attenant au fondouk des Anglais et des Hollandais, est disposé autour d'une cour carrée à galeries, fermée avec une massive porte cochère; il inclut en haut les logements « des négociants, des gens de mer et des voyageurs de passage, les entrepôts et les magasins de vente, mais aussi le logement du consul, la chancellerie, la prison consulaire, la chapelle Saint-Louis et la boulangerie ${ }^{49}$ ». On l'étend en 1678 en annexant un fondouk contigu, qui devient le " fondouk des Négociants (ou des Commerçants) », dans lequel on installe une "Auberge de la Nation " pour l'hébergement des marchands et des marins de passage ${ }^{50}$. Le fondouk marque une frontière juridique et spatiale dans la ville: moyennant un tribut annuel versé par la nation, son consul jouit d'une large autonomie juridictionnelle en matière civile et criminelle, dès qu'il s'agit de juger les litiges survenus entre co-nationaux; de même, les biens acheminés dans le fondouk sont protégés et respectés. Comme au Moyen Âge, les autorités de Tunis essaient de circonscrire les activités des marchands européens à un triangle restreint composé par le fondouk, les quais du port et la douane ${ }^{51}$.

Le fondouk des «nations » étrangères est le résultat d'un compromis pragmatique entre les autorités maghrébines et les communautés de marchands européens, un compromis qui vise à prévenir la conversion des musulmans comme des chrétiens et à maintenir l'ordre public. En cela, le fondouk symbolise tout à la fois la domination des communautés minoritaires mais aussi la négociation de leurs présences : on enferme les habitants du fondouk la nuit, de même qu'on cache à la société tunisoise les tavernes et les célébrations religieuses chrétiennes; le fondouk offre ainsi une solution pour réguler les parcours spatiaux des migrants étrangers, encadrés par les consuls, les chapelains et les résidents co-nationaux de longue durée $e^{52}$. Si les fondouks traduisent, comme à Venise, une domination symbolique de la société d'accueil, et la volonté des autorités de protéger matériellement et spatialement les habitants de toute "infection » ou " contagion ", ils n'empêchent pas les interactions réelles et nombreuses avec le reste de la ville et ses habitants, certes plus difficiles à retracer. Sibylle Backmann, s'appuyant sur les archives notariées et sur les études d'Henry Simonsfeld et d'Ugo Tucci, a par exemple montré qu'il est absurde de considérer le Fondaco dei Tedeschi de Venise comme un lieu imperméable, ou encore un "préservatif urbain» répressif, pour reprendre les mots de Richard Sennett. Les marchands allemands, tout à fait insérés dans l'économie de intus et extra vénitienne, choisissent bien souvent de demeurer dans l'enceinte du Fondaco, ce qui ne les empêche pas de posséder des biens immobiliers à l'extérieur ${ }^{53}$.

Les actes de la chancellerie du consul de France à Tunis permettent d'aboutir à des conclusions sensiblement similaires : outre la présence nombreuse de Génois, de Siciliens et de Corses dans la Régence, qui explique pourquoi l'on parle et l'on écrit principalement l'italien dans le bâtiment, le fondouk des Français est un lieu de rencontres et d'échanges pour les marchands chrétiens, juifs et musulmans, et l'un des espaces centraux des règlements de litiges mercantiles à Tunis. Bien loin de former un isolat hermétique, le fondouk devient l'un des carrefours des transactions commerciales. Pour faciliter le commerce et le rachat des captifs, les fondouks français et anglais jouxtent les nombreux bagnes des quartiers est de la Madîna ; Tunis en compte environ une quinzaine au XVII siècle ${ }^{54}$. Situés en périphérie des quartiers de l'élite, ils constituent des curiosités non seulement pour les voyageurs, qui, dans leurs récits, relatent la dure condition de vie des esclaves, mais ils attirent également les Tunisois, les soldats et les dignitaires turcs. En effet, les bagnes font bien souvent office de tavernes, et le bey accorde des autorisations 
pour ouvrir des débits de boisson: commerce des captifs et commerce des vins vont souvent de pair.

À Livourne, les marchands musulmans ne disposent pas de fondouks qui, comme à Venise, permettraient de les loger et de stocker leurs marchandises; de même, la personnalité juridique d'une hypothétique «nation turque» n'est pas attestée. La présence des musulmans libres est ainsi difficile à cerner et, hormis quelques actes notariés et litiges, peu de documents signalent leur présence dans le port toscan ${ }^{55}$. En revanche, pour les esclaves et les galériens musulmans, dont la présence est mise en scène sur les parois des bâtiments qui donnent sur la darse ${ }^{56}$, la solution du «bagne » a été privilégiée par les Médicis au début du XVII ${ }^{e}$ siècle. Le lieu, qui ressemble en de nombreux points à la structure du fondouk, est voisin de la darse et directement attenant au palais du gouverneur de Livourne, la plus haute autorité de la ville ${ }^{57}$. À Marseille, les esclaves musulmans et les forçats sont spectaculairement visibles: l'arsenal où ils travaillent, en particulier pendant l'hivernage des galères, les expose quotidiennement dans une situation de domination, juste en face des lieux de prestige de la ville ${ }^{58}$. Jean Dumont décrit la misère de la chiourme entassée dans les galères, ajoutant « qu'il ne vient aucune personne de marque à Marseille, que monsieur l'Intendant ne le régale de cette $v^{v u} \mathrm{e}^{59}$ ». Près des galères, et jusqu'au cours Belsunce, certains tiennent de petites échoppes ou offrent leurs services comme domestiques ${ }^{60}$. Lors de l'agrandissement de l'arsenal à la fin des années 1680, des parcelles encloses accueillent un petit cimetière, qui fait également office de lieu de culte pour les esclaves et les musulmans de passage même s'il n'a en aucun cas les apparences d'une mosquée ${ }^{61}$.

La structure du bagne peut apparaître à première vue comme un espace particulièrement caractéristique de la domination. Pourtant, il constitue bien davantage le fruit de compromis et de tractations : il témoigne, en effet, d'une tentative de régulation du phénomène de la captivité, et répond à des exigences sanitaires pour offrir un meilleur logement que dans les galères ${ }^{62}$. Si les bagnes traduisent la rivalité entre musulmans et catholiques à l'époque moderne, leurs existences conjointes à Livourne et Tunis témoignent de règles partagées, basées sur la réciprocité de traitement, qui enjoignent aux autorités locales de permettre l'exercice du culte; d'où la présence de chapelles dans les bagnes de Tunis, et de locaux servant de mosquées dans le bagne de Livourne. Des terrains à l'extérieur des enceintes urbaines furent réservés à l'enterrement des esclaves morts en captivité ; à Livourne, au sud-est des murs de la ville, un terrain vague sert aux enterrements « à la turque " ${ }^{63}$. Ajoutons, en outre, que les bagnes de Livourne et de Tunis ne sont pas des lieux imperméables. S'ils sont, comme les fondouks, fermés la nuit, ils sont traversés le jour par des personnes extérieures, de même que les captifs peuvent vaquer à leurs affaires et exercer des petits métiers dans la ville, voire y louer des pièces ou des échoppes ${ }^{64}$. Le bagne, qui rend particulièrement visible l'esclavage dans les ports, marque également la possibilité d'une inclusion dans les circuits économiques de la ville. À l'époque moderne, il n'est donc pas contradictoire de penser l'espace de la domination comme un espace de la négociation, voire de la protection. En cela, les remarques de Jacques Bottin et de Donatella Calabi à propos des processus de cantonnement sont éclairantes: "c'est peut-être là où elle fut le plus fortement inscrite dans le paysage architectural urbain qu'il est en même temps le plus difficile de dissocier la ségrégation qui s'imposait alors au groupe (en général après négociations) de la protection qui lui était accordée, tantôt par le pouvoir central, tantôt par l'autorité locale ${ }^{65}$ ». Qu'il s'agisse 
de domination, de ségrégation ou de protection, l'espace clos marque l'existence d'un statut juridique collectif qui lui est consubstantiellement associé.

\section{Les « quartiers » juifs}

17 L'existence de «quartiers " juifs pose un autre type de question, à savoir celle du cantonnement communautaire à l'échelle d'une partie de la ville et non du bâtiment. Les années 1670 voient l'établissement à Marseille de petits noyaux de commerçants juifs, à tel point que Colbert écrit à l'intendant Moirant à Aix en 1681 pour lui demander le nombre exact de Juifs et savoir «si ces gens-là sont utiles ou non » dans la ville ${ }^{66}$. Si le port a bien été déclaré «franc » en 1669, les marchands marseillais et la chambre de commerce n'ont jamais vu d'un très bon œil les facilités accordées aux étrangers pour s'y installer, et les manifestations xénophobes n'étaient pas rares, surtout à l'encontre d'étrangers ayant réussi matériellement. Le cas du juif livournais Joseph Vaez Villareal est en cela emblématique : autorisé, suite à la proclamation de l'édit de port franc, à s'installer dans le port en 1670, Villareal développe une entreprise d'import-export avec le Maghreb et le Levant, qui attire d'autres juifs de Livourne. Il subit très vite cependant toute une série d'avanies de la part des échevins et députés du commerce de Marseille, qui vont jusqu'à l'accuser de collusion avec les corsaires d'Alger ${ }^{67}$. Ils ajoutent que «le dit Villareal fut convaincu d'avoir fait une espèce de synagogue dans Sa Maison à Marseille ; tous les Juifs, en nombre de quarante, s'y assemblant pour l'exercice de leur Religion et y célébrant toutes les fêtes et cérémonies sans nulle sorte de retenue ni de ménagement ${ }^{68}$ ". La maison de Villareal est, on le voit, tout à la fois, surveillée et condamnée ; dans l'esprit des échevins de Marseille, « l'espèce de synagogue » brise l'harmonie religieuse et sociale de la ville. Malgré les défenses de Villareal et toute une série de mémoires et d'informations, les Juifs sont expulsés de Marseille en 1682, même si cela n'empêche toutefois pas Abraham Attias, beau-frère de Villareal, d'être autorisé à séjourner temporairement dans le port à partir du 20 septembre $1683^{69}$.

18 À Livourne comme à Tunis, les Juifs sont, au contraire, la minorité religieuse la plus nombreuse. À la fin du XVII e siècle, on estime à 5000 environ le nombre de Juifs à Tunis, dont à peu près 1000 Juifs livournais ${ }^{70}$; le nombre de Juifs à Livourne oscille quant à lui toujours autour des $10 \%$ au XVII ${ }^{\text {e }}$ siècle et on l'estime à 3500 en 1689 , sur une population d'environ 20000 personnes $^{71}$. Le poids démographique des Juifs s'explique notamment par la protection juridique de la communauté: à Livourne, les Livornine de 1593 s'adressent explicitement aux Juifs ainsi qu'aux Marranes chassés de la Péninsule ibérique et prévoient de leur octroyer une large autonomie juridictionnelle. A Tunis, les Juifs tunisiens (Twânsa), établis de longue date, et livournais (Grâna), d'immigration récente, bénéficient eux aussi d'une large autonomie administrative ainsi que d'une réelle liberté de culte. Certes moins protégés qu'en Toscane, les Juifs de Tunis payent un impôt (jazya) par capitation que répartit le chef de la communauté, le caïd des juifs, qui sert d'intermédiaire avec les autorités locales. Liberté de culte et protection juridique aboutissent à la création de "quartiers ", organisés de manière fonctionnelle pour les besoins rituels (synagogue, bains, établissements kasher, écoles, cimetières à proximité). À Livourne, ce quartier occupe la partie sud de la ville, derrière la cathédrale, près du Bastione del Casone. S'il n'y a pas de portes, l'habitat des Juifs est tout de même circonscrit, dès la première moitié $\mathrm{du} X V \mathrm{XI}^{\mathrm{e}}$ siècle, à une partie de la ville dessinée par la Via Reale d'un côté, et la Via Serristori de l'autre, interdisant semble-t-il la prestigieuse Via 
Ferdinanda ${ }^{72}$. Les noms de rue sont explicites : "Quattro Canti degli Ebrei », «Via Santa Maria degli Ebrei », «Via della Sinagoga ${ }^{73}$ ». Les récits de voyage ne manquent quasiment jamais de décrire le quartier juif, et lui accolent souvent le nom de ghetto. Pourtant, à la différence de Venise, les Juifs de Livourne ne portent aucune marque vestimentaire distinctive, et ne sont ni enfermés, ni soumis à des interdits nocturnes; au-delà, on achète, on loue et on réside à l'extérieur de ses limites, à l'instar d'Abraham Ergas qui achète une maison neuve et très grande via Ferdinanda ${ }^{74}$. Renzo Toaff montre même que sur 207 foyers en 1645, 136 vivent dans les rues du " ghetto », et 16 sur la via Ferdinanda ${ }^{75}$ . Sur cette longue rue qui traverse Livourne d'ouest en est, plusieurs immeubles sont conjointement habités par des Juifs et des non-Juifs ${ }^{76}$. À Tunis, qu'il s'agisse de Juifs tunisois ou livournais, le quartier juif est celui de la Hâra, qui remonte vraisemblablement à la période almohade, et qui s'agrandit considérablement aux XVII ${ }^{\mathrm{e}}$ et XVIII ${ }^{\mathrm{e}}$ siècles $^{77}$. Sis au nord de la Madîna, il est délimité au nord-ouest par la rue des Djerbiens et au sud par la rue Sîdî Srîdîa. On y trouve la Grande synagogue, ainsi que plusieurs souks (dont le sûq Grâna), ainsi qu'un bagne ${ }^{78}$. S'il n'y a pas d'enceinte à proprement parler qui enveloppe le quartier - ce qui explique son extension à l'époque moderne -, la Hâra a des portes, comme le quartier des souks ${ }^{79}$. Il semble cependant qu'une partie des Juifs les plus aisés ait tôt cherché à s'installer en dehors de la Hâra, en se rendant plus au sud vers le quartier franc.

Ces deux quartiers juifs laissent entrevoir quelques similitudes, que l'on peut mettre en regard avec les "caractères récurrents" pointés par Donatella Calabi à propos des ghettos italiens du XVI ${ }^{\mathrm{e}}$ siècle ${ }^{80}$. Tout d'abord, on remarque l'inclusion des quartiers juifs dans le tissu urbain, situés à Livourne comme à Tunis à l'intérieur des murailles de la ville ; cette centralité du "quartier juif » est également, à l'exception de Venise et de Palerme, une caractéristique des ghettos juifs italiens, souvent installés près des zones d'activité commerciale. Comme pour les fondouks, le quartier n'est pas hermétique. Les Juifs, à Tunis et à Livourne, participent à l'artisanat local comme au grand commerce et se déplacent en ville, de même que les locaux et les autres étrangers peuvent se rendre dans le quartier juif - et notamment dans le sûq Grâna - pour vaquer à leurs affaires. À Livourne, des Italiens de modeste condition travaillent au Casone, employés chez des diamantaires ou dans la fabrique de corail. Si la présence juive à Tunis est fort ancienne, les migrations de Juifs andalous puis livournais aux $\mathrm{XVI}^{\mathrm{e}}$ et $\mathrm{XVII}^{\mathrm{e}}$ siècles ont amplement reconfiguré l'organisation de la Hâra, devenue l'un des centres du commerce international dans la Régence. À Livourne, les Médicis souhaitent attirer les marchands et les courtiers sépharades pour ouvrir de nouvelles routes de commerce avec la ville nouvelle, faisant du quartier juif un lieu essentiel du négoce en Toscane. La langue, en revanche, peut signaler les limites du «quartier »: dans le Casone, un voyageur entend surtout parler portugais, si l'on en croit le père Labat ${ }^{81}$. Mais ce qui interpelle davantage à Tunis, comme dans les ghettos italiens, c'est bien la contrainte vestimentaire et le couvrechef noir que les Juifs sont contraints de porter (chéchia pour les Twânsa et chapeau «à l'européenne » pour les Grâna).

20 Ces quartiers sont, en outre, caractérisés par une densité de population très élevée ${ }^{82}$. À Tunis, l'essor démographique de la communauté juive entraîne « un surpeuplement de la Hâra, une crise de logement et une hausse des loyers qui contraignent la communauté juive à adopter une réglementation protégeant les locataires acquittant leur loyer contre une éventuelle éviction ${ }^{83}$ ". Dans les ghettos italiens, cette densification progressive de la population contribue à une « augmentation de volume, un accroissement du nombre des 
étages et des parties adjonctives [...], une multiplication des subdivisions internes, le tout justifié par les limites rigides imposées au périmètre de la zone ${ }^{84} »$. Si ce phénomène concerne bien les Juifs pauvres à Tunis et Livourne, il ne touche toutefois pas les familles plus aisées de la communauté qui peuvent louer et acheter en dehors de la zone d'abord dévolue à leur installation. La fin du XVII siècle est d'ailleurs caractérisée par une expansion de l'habitat juif à Livourne et Tunis. André Raymond rappelle que la superficie de la Hâra s'est étendue sur 2,3 hectares, à l'est des 4 hectares initiaux de la zone hafside ${ }^{85}$ . À Livourne, le père Labat, au début du XVIII ${ }^{\mathrm{e}}$ siècle, note dans sa description du quartier juif qui n'est pas exempte de topoï antisémites : «[les Juifs] sont logés aussi au large qu'il leur plait. Ils étendent leur quartier tant qu'ils veulent. On se plaignait en 1716 quand je passai par cette Ville, que leur nombre croissait à vue d'œil, qu'ils louaient des maisons qui n'avaient jamais été habitées que par des Chrétiens, et que si le Prince n'y mettait ordre, ils rempliraient bientôt toute la Ville ${ }^{86}$ ". S'il faut se méfier de la relation du père Labat (qui estime à 22000 le nombre de Juifs à Livourne en 1710 !), celle-ci traduit toutefois une réalité, à savoir la possibilité pour certains Juifs, souvent aisés, de s'installer dans d'autres quartiers : la via dell'Amore et la via della Pallacorda alla Francese (au niveau de l'actuelle via Santa Fortunata), perpendiculaires à la via Ferdinanda, concentrent progressivement une forte proportion de Juifs ${ }^{87}$. L'extension de l'habitat juif dans les espaces clôturés italiens, où le patrimoine immobilier juif est dans les mains des autorités locales, semble interdire, en revanche, ce franchissement de limites: on ne concède qu'aux Juifs dans les ghettos le pouvoir de transformer leur habitation, ce qui explique l'ajout progressif d'étages. Notons enfin que des musulmans possèdent des maisons qu'ils louent aux Juifs dans la Hâra, de même qu'à Livourne, des chrétiens sont propriétaires d'immeubles dans les rues du Casone ${ }^{88}$.

21 Dans les ghettos italiens, « les immeubles d'habitation qui abritaient la population juive [...] restent précaires et de qualité médiocre ${ }^{89} »$. Les investissements dans les ghettos portent surtout sur l'intérieur des habitations. Si cela semble également le cas dans la Hâra tunisoise, en raison notamment de la poussée démographique du XVII siècle, l'extension vers l'est permet également la construction ou l'achat de bâtiments plus spacieux et confortables. L'est de la Hâra devenant l'un des lieux privilégiés de la spéculation immobilière au XVII ${ }^{e}$ siècle, les beys de Tunis louent aux négociants juifs livournais maisons et magasins (makhzan) construits sur des parcelles restaurées ${ }^{90}$. L'habitat des Juifs à Livourne varie en fonction du niveau social : des Juifs pauvres ne peuvent louer qu'une petite chambre, quand un Rafael de Luna, notable de la communauté, est en 1644 propriétaire d'un appartement de neuf pièces Via della Pallacorda alla Francese ${ }^{91}$. Le même père Labat note, malgré sa répugnance pour le quartier juif qu'il juge sale, que « les maisons y sont belles [...] et [...] la plûpart [...] trèsbien meublées ${ }^{92} »$. Enfin, ces quartiers concentrent tous les services communautaires, civils et religieux - la synagogue jouant bien entendu le rôle de pôle centrifuge. Sur ce point, il ne semble guère y avoir de différence entre le « quartier » et le "ghetto ». Les ghettos italiens ont, en effet, « un équipement en services collectifs supérieur à celui des autres quartiers » de la ville ${ }^{93}$. On peut toutefois émettre l'hypothèse d'une différence sensible : la diffusion juridiquement possible de l'habitat des Juifs à Livourne et Tunis, et la concentration des Juifs pauvres dans la limite impartie à l'origine par les autorités locales, rendent moins importants les efforts de voirie dans les « quartiers » que dans les ghettos, où l'enfermement a contraint la communauté à adopter des mesures hygiéniques strictes. 

explique leur poids démographique et l'extension de leurs quartiers : pour les premiers, les Livornine, et pour les seconds le statut de dhimmi, ont permis le libre exercice de la religion et une relative autonomie communautaire. Si des formes de domination juridique et symbolique variées subsistent à l'encontre des Juifs, le quartier est le résultat d'un regroupement fonctionnel et pragmatique qui n'interdit pas la diffusion ni de multiples interactions avec les autres parties de la ville. Espace clos, fondouks, bagnes et quartiers juifs traduisent l'existence d'un statut juridique, d'une négociation de droits entre autorités locales et minorités, même si les divers statuts sociaux au sein de la minorité, et la différence d'histoires et de parcours migratoires (entre Twânsa et Grâna par exemple) produisent de multiples formes d'appropriation du territoire urbain. La création d'un statut qui définit droits et obligations de la communauté a donc des manifestations spatiales: au lieu de culte des minorités religieuses s'ajoute un régime d'installation dérogatoire qui a des incidences sur le bâti.

De ces trois formes de territorialisation et de visibilité de la présence étrangère, il ressort que les espaces des minorités traduisent des possibilités sociojuridiques généralement reconnues par les statuts, les traités ou les lois lorsqu'elles sont matérialisées par des espaces - collectifs et individuels - négociés et plus ou moins précisément délimités ; en revanche, il serait sans doute incorrect de déduire d'un relatif défaut de visibilité topographique des minorités des impossibilités juridiques d'inclusion. En effet, les nombreuses stratégies de contournements et de dérogations à l'œuvre ont généralement peu de prises sur la morphologie urbaine, tant elles participent plutôt d'une logique d'adéquation et d'homogénéisation avec la ville. La seule visibilité accordée aux étrangers ne permet certes pas de décliner le large éventail des degrés d'extranéité qui pouvaient exister à l'époque moderne, mais elle signale, en revanche, des marges de manœuvre matérielles et territoriales, qui complètent l'étude sociojuridique des processus d'inclusion.

\section{NOTES}

1. Julius KIRSCHNER et Laurent MAYALI (éd.), Privileges and Rights of Citizenship: Law and the Juridical Construction of Civil Society, Berkeley, Robbins collection publications, 2002, 297 p.; Tamar HERzoG, Defining Nations : Immigrants and Citizens in Early Modern Spain and Spanish America, New Haven, Yale University Press, 2003, 325 p.; Peter SAHLINS, Unnaturally French: Foreign Citizens in the Old Regime and after, Ithaca-Londres, Cornell University Press, 2004, 454 p.; Simona CERUTTI, "À qui appartiennent les biens qui n'appartiennent à personne? Citoyenneté et droit d'aubaine à l'époque moderne ", Annales, Histoire, Sciences Sociales, n 62/2, 2007, p. 355-383 ; Hanna SONKAJÄRVI , Qu'est-ce qu'un étranger? Frontières et Identifications à Strasbourg, 1681-1789, Strasbourg, Presses universitaires de Strasbourg, 2008, $223 \mathrm{p}$.

2. Jacques BOTTIN et Donatella CALABI (éd.), Les étrangers dans la ville. Minorités et espace urbain du bas Moyen Âge à l'époque moderne, Paris, Editions de la Maison des sciences de l'homme, 1999, 486 p.; Denis MENJOT et Jean-Luc PINOL (éd.), Les immigrants et la ville: insertion, intégration, discrimination (XII ${ }^{e}$-XX ${ }^{e}$ siècles), Paris, L'Harmattan, 1996, 230 p.

Liame, 24 | 2012 
3. «L'étranger ", Recueils de la société Jean Bodin pour l'histoire comparative des institutions, 9-10, Bruxelles, 1958 ; Pilar GONZÁLEZ-BERNALDO, Manuela MARTINI, Marie-Louise KAPLAN (éd.), Étrangers et sociétés : représentations, coexistences, interactions dans la longue durée, Rennes, PUR, 2008, 482 p. (en particulier les articles de Neithard BULST, «Les étrangers dans la ville. Perception de l'« autre » et rapport avec « autrui » selon le droit municipal allemand au Moyen-Âge », p. 45-62 et Wolfgang KAISER, « Extranéités urbaines à l'époque moderne », p. 77-86).

4. Bernard LEPETIT, « Proposition et avertissement », dans J. Bottin et D. Calabi (éd.) Les étrangers dans la ville... op. cit., p. 11-15 (ici p. 13).

5. Maurice AYMARD, «Introduction », dans J. Bottin et D. Calabi (éd.), Les étrangers dans la ville... op. cit., p. 19-23 (ici p. 21).

6. Peregrine HORDEN et Nicholas PURCELL, The Corrupting Sea. A Study of Mediterranean History, Oxford, Blackwell, 2000, p. 101.

7. Jocelyne DAKHLIA, «La question des lieux communs. Des modèles de souveraineté dans l'Islam méditerranéen ", dans Bernard Lepetit (éd.), Les formes de l'expérience. Une autre histoire sociale, Paris, Albin Michel, 1995, p. 39-61 (ici p. 45 et sqq.).

8. Sur cet espace commercial, voir Sadok BOUBAKER, La Régence de Tunis au XVII ${ }^{e}$ siècle : ses relations commerciales avec les ports de l'Europe méditerranéenne, Marseille et Livourne, Zaghouan, Ceroma, 1987, 248 p. ; Id., " Négoce et enrichissement individuel à Tunis du XVII ${ }^{\mathrm{e}}$ siècle au début du XIX ${ }^{\mathrm{e}}$ siècle ", Revue d'Histoire moderne et contemporaine, 50/4 (2003), p. 29-62 (ici carte p. 33) ; Christian WINDLER, La diplomatie comme expérience de l'autre. Consuls français au Maghreb (1700-1840), Genève, Droz, 2002, p. 18.

9. Sadok BOUBAKER, La Régence de Tunis... op. cit., p. 83 ; Paul SEBAG, Tunis: Histoire d'une ville, Paris, L'Harmattan, 1998, p. 236.

10. Donatella CALABI, Il mercato e la città : piazze, strade, architetture d'Europa in età moderna, Venise, Marsilio, 1993, p. 30.

11. Wolfgang KAISER et Claudia MOATTI, "Introduction" dans id. (éd.), Gens de passage en Méditerranée de l'Antiquité à l'époque moderne: procédures de contrôle et d'identification, Paris, Maisonneuve et Larose/MMSH, 1997, p. 9-21.

12. Sadok BOUBAKER, La Régence de Tunis... op. cit., p. 62.

13. Antoine de RUFFI et Louis Antoine de RUFFI, Histoire de la ville de Marseille contenant tout ce qui s'y est passé de plus mémorables depuis sa fondation..., Marseille, Henri Martel, 1696, vol. 2, Livre X, p. 54. Voir également: Wolfgang KAISER, Marseille au temps des troubles. Morphologie sociale et luttes de factions, 1559-1596, Paris, EHESS, 1992, p. 81.

14. Jacques REVAult, Le fondouk des Français et les consuls de France à Tunis (1660-1860), Paris, Ed. Recherche sur les civilisations, 1984, p. 12.

15. André RAYMOND, Tunis sous les Mouradites. La ville et ses habitants au XVII ème siècle, Tunis, Cérès éd., 2006, p. 245-246.

16. Laurent d'ARVIEUX, Mémoires du chevalier d'Arvieux, envoyé extraordinaire du Roy à la Porte, consul d'Alep, d'Alger, de Tripoli et autres Échelles du Levant... mis en ordre par le R. P. Jean-Baptiste Labat, Paris, Delespine, 1735, vol. 4, p. 4.

17. Sur les Juifs de Tunis: Hayyim Ze'ev-Joachim W. HIRSCHBERG, A History of the Jews in North Africa: From the Ottoman Conquests to the Present Time, Leyde, E. J. Brill, 1981, p. 90-146; Haïm ZAFRANI, Juifs d'Andalousie et du Maghreb, Paris, Maisonneuve et Larose, 1996, 437 p.; Paul SEBAG, Histoire des juifs de Tunisie, des origines à nos jours, Paris, L'Harmattan, 1991, 335 p. Sur les Morisques : John Derek LATHAM, From Muslim Spain to Barbary: Studies in the History and Culture of the Muslim West, Londres, Variorum reprints, 1986, 348 p. ; Miguel de EPALZA, Los Moriscos antes $y$ después de la expulsión, Madrid, Mapfre, 1992, 312 p.; Aïssa LOTFI, «Dans la tourmente de l'exil : plaidoirie d'un morisque de Murcie installé à Tunis au XVII ${ }^{\mathrm{e}}$ siècle ", Cahiers de la Méditerranée, 79 (2009), p. 337-349. Sur la présence européenne - en particulier française, mais aussi corse et 
italienne - dans la Tunis du XVII ${ }^{\mathrm{e}}$ siècle, les actes de la chancellerie des consuls de France sont tout à fait éclairants : Pierre GRANDCHAMP, La France en Tunisie. Documents inédits (1582-1705), Tunis, J. Aloccio, 1921-1933, 10 vol. Sur le brassage des populations à Tunis, voir André RAYMOND, Tunis sous les Mouradites... op. cit., p. 53-171; Taoufik BACHROUCH, Formation sociale barbaresque et pouvoir à Tunis au XVII siècle, Tunis, Université de Tunis, 1977, p. 33-44.

18. Dario MATTEOnI, Livorno, Rome-Bari, Laterza, 1985, p. 15-21; Lucia FRATTARELli FISCHER, «Livorno città nuova 1574-1609 ", Società e Storia, 46 (1989), p. 873-893. En cela, Livourne se distingue nettement de Marseille et de Tunis, où les voyageurs ont plutôt tendance à noter l'étroitesse des rues.

19. Lucia frattarelli fischer et Paolo castignoli (éd.), Le 'Livornine' del 1591 e 1593, Livourne, Cooperativa Risorgimento, 1987, $23 \mathrm{p}$.

20. Lucia FRATTARELLI FISCHER, « Livorno città nuova... art. cit. », p. 890.

21. Rita MAZZEI, «L'economia pisana e la dinamica del commercio internazionale nell'età moderna ", dans Marco TANGHERONI (éd.), Pisa e il Mediterraneo. Uomini, merci, idee dagli Etruschi ai Medici, Milan, Skira, 2003, p. 293-297 (ici p. 296).

22. Vincenzo PITTI, «Descrizione di Pisa fatta da Vincenzo Pitti l'anno 1616 », [En ligne : http:// dante.di.unipi.it/ricerca/html/dpp.html], p. 102.

23. Jean Dumont, Voyages de Mr. Du Mont en France, en Italie, en Allemagne, à Malthe et en Turquie. Contenant les recherches \& observations curieuses qu'il a faites en tous ces païs..., La Haye, E. Foulque, 1699, vol. I, p. 159-160. À titre indicatif, notons que 343 «étrangers » furent enregistrés à Marseille de 1697 à 1703 par l'intendance de Provence, dont une très grande majorité (293) originaire du comté de Nice: Jean-François DUBost et Peter SAHLINS, Et si on faisait payer les étrangers : Louis XIV, les immigrés et quelques autres, Paris, Flammarion, 1999, p. 248.

24. Lewes ROBERTS, The Merchants mappe of commerce..., Londres, R. Mabb, 1638, chap. III, p. 25.

25. Ibid., chap. CLXXIII, p. 90.

26. Wolfgang KAISER, Marseille au temps des troubles... op. cit., p. 353.

27. Jacques SAVARY DES BRUSLONS, Dictionnaire Universel de Commerce..., Paris, J. Estienne, 1723, vol. II, col. 1186 .

28. Jean-Joseph JULIEN, Nouveau Commentaire sur les Statuts de Provence, Aix, Esprit David, 1778, vol. I, p. 40-42.

29. Jacques воттіN et Donatella CALABI, «Introduction » dans id. (éd.), Les étrangers dans la ville... op. cit., p. 1-9 (ici p. 6).

30. Archives de la Chambre de Commerce et d'Industrie de Marseille, $\mathrm{K}$ 50, Lettres de François Cotolendy, 23 juillet 1686, non foliotée.

31. Archivio di Stato di Livorno (désormais ASL), Decima, 30 « Copia del estimo vecchio dell'anno 1559 ».

32. Sadok BOUBAKER, « Négoce et enrichissement... art. cit. », p. 37.

33. Francesca FUNIS, "Gli insediamenti dei Greci a Livorno tra Cinquecento e Seicento ", Città $e$ storia, n spécial «La città cosmopolita», 2/1 (2007), p. 61-75 (ici p. 70), à partir de ASL, Governatore ed Auditore, "Suppliche », 2602/II, fo 302. Voir également ASL, Decima, 30, f० 190 et $\mathrm{f}^{\circ}$ 169.

34. ASL, Decima, 30, fo 190 , fo 220 , fo $268, f^{\circ} 306$.

35. Jean-François DUBost, «Les Italiens dans les villes françaises: $\mathrm{XVI}^{\mathrm{e}}-\mathrm{XVII}{ }^{\mathrm{e}}$ siècles », dans D. Menjot et J.-L. Pinol (éd.), Les immigrants et la ville... op. cit., p. 91-106 (ici p. 92).

36. Wolfgang KAISER, «Récits d'espace. Présence et parcours d'étrangers à Marseille au XVI siècle ", dans J. Bottin et D. Calabi (éd.), Les étrangers dans la ville... op. cit., p. 299-312 (ici p. 309).

37. Wolfgang KAISER, Marseille au temps des troubles... op. cit., p. 30.

38. Jean-François DUBoST, « Les Italiens dans les villes françaises... art. cit. », p. 100.

39. Wolfgang KAISER, « Récits d'espaces... art. cit. », p. 310. 
40. Raymond H. KÉVORKIAN, "Le négoce international des Arméniens au XVII ${ }^{\mathrm{e}}$ siècle ", dans Arménie entre Orient et Occident, trois mille ans de civilisation, Paris, BnF, 1996, p. 142-151 (ici p. 148).

41. Sur les Arméniens de Marseille: Olivier RAveuX, «Entre appartenance communautaire et intégration locale: la colonie marseillaise des marchands arméniens de la Nouvelle-Djoulfa (Ispahan), 1669-1695 ", article encore non publié.

42. Stefano VILLANI, “'Cum scandalo catholicorum...'. La presenza a Livorno di predicatori protestanti inglesi tra il 1644 e il $1670 »$, Nuovi Studi Livornesi, VII (1999), p. 9-58.

43. Lucia FRATTARELli FISCHER, "Lo sviluppo di una città portuale : Livorno, 1585-1720 ", dans Marco folin (éd.), Sistole/Diastole: Episodi di trasformazione urbana nell'Italia delle città, Venise, Istituto veneto di scienze, lettere ed arti, 2006, p. 271-333 (ici p. 303 et 305) ; Francesca TRIVELLATo , The Familiarity of Strangers: The Sephardic Diaspora, Livorno and Cross-Cultural Trade in the Early Modern Period, New Haven, Yale University Press, 2009, p. 80-81.

44. Stefano VILLANI, «L'histoire religieuse de la communauté anglaise de Livourne (XVII ${ }^{\mathrm{e}}$ et XVIII ${ }^{\mathrm{e}}$ siècles) ", dans Albrecht BURKARDT (éd.), Commerce, voyage et expérience religieuse, XVI ${ }^{e}-X V I I I^{e}$ siècles, Rennes, PUR, 2007, p. 257-274 (ici p. 262).

45. Francesca TRIVELLATO, The Familiarity of Strangers... op. cit., p. 81.

46. Daniele PESCIATINI, "Il 'Celebì' del Bagno Turco », dans Gli Armeni lungo le strade d'Italia, PiseRome, Ist. Editoriali e Poligrafici, 1998, p. 73-101.

47. Sadok BOUBAKER, La Régence de Tunis... op. cit., p. 141 ; Jacques REVAULt, Le fondouk des Français... op.cit.; André RAYMOND, Tunis sous les Mouradites...op.cit., p. 246; Ahmed SAADAOUI, «Les Européens à Tunis aux XVII ${ }^{\mathrm{e}}$ et XVIII ${ }^{\mathrm{e}}$ siècles », Cahiers de la Méditerranée, 67 (2003), p. 61-84.

48. Dominique VALÉRIAN, «Les marchands latins dans les ports musulmans méditerranéens : une minorité confinée dans des espaces communautaires", Revue du Monde Musulmans et de la Méditerranée, 107-110 (2005), p. 437-458; Id. «Le fondouk, instrument du contrôle sultanien sur les marchands étrangers dans les ports musulmans (XII $-\mathrm{XV}^{\mathrm{e}}$ siècle) ?", dans Claudia MOATTI (éd.), La mobilité des personnes en Méditerranée, de l'Antiquité à l'époque moderne. Procédures de contrôle et documents d'identification, Rome, EFR, 2004, p. 677-698.

49. Christian WINDLER, «La révolution française et l'espace urbain d'une échelle du Maghreb : le cas de Tunis", dans J. Bottin et D. Calabi (éd.), Les étrangers dans la ville... op. cit., p. 77-86 (ici p. 77) ; Jacques REVAULT, Le fondouk des Français... op. cit., p. 21.

50. Pierre GRANDChAmp, "Établissement en 1692 d'une auberge dans le Fondouk de la nation française. Sa suppression en $1778 »$, Revue tunisienne, 25 (1918), p. 226-232.

51. Dominique VALÉRIAN, « Les marchands latins dans les ports musulmans... art. cit. », p. 447.

52. Les résidents de longue durée n'étaient vraisemblablement guère nombreux (Sadok BOUBAKER, La Régence de Tunis... op. cit., p. 142).

53. Sybille BACKMANN, "Le Fondaco dei Tedeschi à Venise. Exclusion et inclusion des marchands allemands dans l'économie vénitienne (1550-1650) », dans Wolfgang KAISER (éd.), Entre funduq et loggia, à paraître.

54. André RAYMOND, Tunis sous les Mouradites... op. cit., p. 128-136.

55. Guillaume CALAFAT et Cesare SANTUS, «Les avatars du 'Turc'. Esclaves et commerçants musulmans en Toscane (1600-1750)», dans Jocelyne DAKHLIA et Bernard VINCENT (dir.), Les Musulmans dans l'histoire de l'Europe, tome I : Une intégration invisible, Paris, Albin Michel, 2011, p. 471-522.

56. Lucia FRATTARELLI FISCHER, « Lo sviluppo di una città ... art. cit. », p. 285.

57. Lucia FRATTARELLI FISCHER, «Il bagno delle galere in 'terra cristiana'. Schiavi a Livorno fra Cinque e Seicento ", Nuovi Studi Livornesi, VIII (2000), p. 69-94.

58. Jocelyne DAKHLIA, "Musulmans en France et en Grande-Bretagne à l'époque moderne: exemplaires et invisibles", dans J. Dakhlia et B. Vincent (éd.), Les Musulmans dans l'histoire de l'Europe... op. cit., p. 231-413 (ici p. 282-311). Voir également, pour de nombreux plans et cartes : 
André ZYSBERG, Marseille au temps du Roi-Soleil : la ville, les galères, l'arsenal, Marseille, J. Laffitte, 2007, p. 102-183.

59. Jean Dumont, Voyages de Mr. Du Mont... op. cit., vol. I, p. 162.

60. André ZYSBERG, Les galériens. Vies et destins de 60000 forçats sur les galères de France, 1680-1748, Paris, Le Seuil, 1987, p. 148-151.

61. Régis BERTRAND, "Les cimetières des 'esclaves turcs' des arsenaux de Marseille et de Toulon au XVIII siècle ", Revue des mondes musulmans et de la Méditerranée, 99-100 (2002), p. 205-217 (ici p. 207-209).

62. André ZYSBERG, Les galériens... op. cit., p. 126-161.

63. Stefano VILLANI, «Alcune note sulle recinzioni dei cimiteri acattolici livornesi ", Nuovi Studi Livornesi, 11 (2004), p. 35-51 (ici p. 46); Guillaume CALAFAT et Cesare SANTUS, «Les avatars du 'Turc'... art. cit. », p. 493.

64. Guillaume CALAFAT et Cesare SANTUS, «Les avatars du 'Turc'... art. cit. », p. 486-492.

65. Jacques BOTTIN et Donatella CALABI, «Introduction... art. cit. », p. 5.

66. Pierre CLÉMENT, Lettres, Instructions et Mémoires de Colbert..., Paris, Imprimerie impériale, 1863, vol. II, p. 722.

67. Adolphe CRÉMIEUX, « Un établissement juif à Marseille au XVII siècle », Revue des études juives, LV (1908), p. 119-145 et LVI (1908), p. 99-123.

68. Bibliothèque nationale de France, Ms. Français, 18979, fo 146 ; document également transcrit dans Adolphe CRÉMIEUX, « Un établissement juif... art. cit. », LVI, p. 121.

69. Archives Nationales, fonds de la Marine, $\mathrm{B} / 3 / 44, \mathrm{f}^{\circ} 42$.

70. André RAYMOND, Tunis sous les Mouradites... op. cit., p. 148. Les estimations sur la population totale de la ville varient de manière considérable. Taoufik Bachrouch, à la suite de Robert Brunschvig, a proposé le seuil maximal de 100000 personnes pour l'époque moderne (Taoufik Bachrouch, Formation sociale barbaresque... op. cit., p. 25-32).

71. Lucia FRATTARELLI FISCHER, "Proprietà e insediamento ebraici a Livorno dalla fine del Cinquecento alla seconda metà del Settecento ", Quaderni Storici, 54 (1983), p. 879-896.

72. Giuseppe vivoli, Annali di Livorno della Sua Origine sino All'Anno di Gesu' Cristo 1840, Livourne, Bastogi, 1846, vol. IV, p. 153.

73. Renzo TOAFF, La nazione ebrea a Livorno e Pisa (1591-1700), Florence, L. S. Olschki, 1990, p. 136.

74. Francesca TRIVELlato, The Familiarity of Strangers... op. cit., p. 88.

75. Renzo TOAFF, La nazione ebrea... op. cit., p. 137.

76. ASL, Decima, 30, fo 292 , fo 309.

77. Paul SEBAG, La Hara de Tunis : l'évolution d'un ghetto nord-africain, Paris, PUF, 1959, 202 p.

78. André RAYMOND, Tunis sous les Mouradites... op. cit., p. 334.

79. Paul SEBAG, Tunis, histoire d'une ville... op. cit., p. 274.

80. Donatella CALABI, "La cité des juifs en Italie entre $\mathrm{XV}^{\mathrm{e}}$ et $\mathrm{XVI}{ }^{\mathrm{e}}$ siècle », dans J. Bottin et $\mathrm{D}$. Calabi (éd.), Les étrangers dans la ville... op. cit., p. 25-40 (ici p. 37-40).

81. Jean-Baptiste LABAT, Voyages du P. Labat... en Espagne et en Italie, Paris, Delespine, 1730, vol. II, p. 135.

82. Paul SEBAG, Tunis : histoire d'une ville... op. cit., p. 165 ; André RAYMOND, Tunis sous les Mouradites... op.cit., p. 241-242; Renzo TOAFF, La nazione ebrea... op. cit.; sur la démographie du ghetto de Venise: Giovanni FAVERo et Francesca tRIVELlato, "Gli abitanti del ghetto di Venezia in età moderna : dati e ipotesi ", Zakhor. Rivista di storia degli ebrei d'Italia, VII (2004), p. 9-50.

83. André RAYMOND, Tunis sous les Mouradites... op. cit., p. 242.

84. Donatella CALABI, « La cité des juifs... art. cit. », p. 39.

85. André RAYMOND, Tunis sous les Mouradites... op. cit., p. 242.

86. Jean-Baptiste LABAT, Voyages du P. Labat... op. cit., vol. II, p. 134. 
87. Renzo toAfF, La nazione ebrea... op. cit., p. 137.

88. Par exemple : ASL, Decima, $30, \mathrm{f}^{\circ} 304 \mathrm{v}^{\circ}$.

89. Donatella CALABI, « La cité des juifs... art. cit. », p. 39

90. André RAYMOND, Tunis sous les Mouradites... op. cit., p. 239.

91. Renzo TOAFF, La nazione ebrea... op. cit., p. 448.

92. Jean-Baptiste LABAT, Voyages du P. Labat... op. cit., vol. II, p. 133-134.

93. Donatella CALABI, « La cité des juifs... art. cit. », p. 38.

\section{RÉSUMÉS}

Cet article cherche à décrire les différentes formes de territorialisation et de visibilité des "étrangers" et des "minorités» dans trois ports méditerranéens du XVII ${ }^{\mathrm{e}}$ siècle reliés commercialement : Livourne, Tunis et Marseille. Il s'intéresse tout d'abord aux espaces des «nations », peu apparents dans la Marseille de l'époque moderne et, à l'inverse, très visibles à Tunis et Livourne sous forme de "quartiers" ou d'espaces clos (fondouks). Une attention particulière est ensuite portée aux bagnes des esclaves et des galériens dans les trois villes, ainsi qu'aux dissemblances et similitudes des quartiers juifs de Livourne et de Tunis. L'étude de la morphologie urbaine est présentée ici à la fois comme un révélateur potentiel et comme un complément d'analyse utile à l'histoire des processus sociojuridiques d'inclusion ou d'exclusion des étrangers dans les villes de l'époque moderne.

« Minorities» Topographies: notes about Livorno, Tunis and Marseilles in the XVII ${ }^{\text {th }}$ century.

This essay aims at describing different forms of territorialization and visibility of "foreigners" and "minorities" in three ports tightly linked by trade in XVII ${ }^{\text {th }}$ century Mediterranean Sea: Livorno, Tunis and Marseilles. It firstly focuses on the spaces of the "nations", scarcely apparent in Early modern Marseilles, but very visible in Tunis and Livorno in the form of "districts" or closed spaces (funduqs). It then concentrates on slaves and galley-slaves prisons in those cities, and on differences and similarities between Livorno's and Tunis' Jewish districts. By using urban morphological analysis, one's intention is to prove its usefulness for the comprehension of the history of socio-legal processes of inclusion and exclusion of foreigners and minorities in Early-Modern cities.

\section{INDEX}

Mots-clés : étrangers, Juifs, minorités, quartier, ghettos, bagnes, Livourne, Tunis, Marseille

Keywords : foreigners, Jews, minorities, district, ghettos, slave prisons, Livorno, Tunis, Marseille

\section{AUTEUR}

\section{GUILLAUME CALAFAT}

Université Paris I-Panthéon Sorbonne

Ecole Française de Rome 